Cite as: Stephane H Maes, (2020), "Gravity Stabilizes Electroweak Vacuum - No Bubble of Nothing to Worry About!", viXra:2007.0173v1, https://vixra.org/pdf/2007.0173v1.pdf, https://

shmaesphysics.wordpress.com/2020/06/24/gravity-stabilizes-electroweak-vacuum-no-bubble-of-nothing-toworry-about/, June 24, 2020.

\title{
Gravity Stabilizes Electroweak Vacuum - No Bubble of Nothing to Worry About!
}

\author{
Stephane H. Maes ${ }^{1}$
}

June 24, 2020

\begin{abstract}
:
In a multi-fold universe, gravity emerges from Entanglement through the multi-fold mechanisms. As a result, gravity-like effects appear in between entangled particles that they be real or virtual. Long range, massless gravity results from entanglement of massless virtual particles. Entanglement of massive virtual particles leads to massive gravity contributions at very smalls scales. Multi-folds mechanisms also result into a spacetime that is discrete, with a random walk fractal structure and non-commutative geometry that is Lorentz invariant and where spacetime nodes and particles can be modeled with microscopic black holes. All these recover General relativity at large scales and semi-classical model remain valid till smaller scale than usually expected. Gravity can therefore be added to the Standard Model. This can contribute to resolving several open issues with the Standard Model.

Since the work of Coleman, it is known that vacuum may be false in the universe with truer vacuum reachable by quantum tunneling. With the discovery and analysis of the Higgs boson, it has been estimated that, with the mass of the Higgs boson, our universe is right on the stable side, but the edge of instability; playing the fire, or rather the risk of a bubble of nothing erasing everything in the universe. In this paper we show that by adding non-negligible gravity to the standard model, the universe can move further away from the brink of instability; a reassuring thought. This satisfying result help us further argue for systematically considering adding gravity to the Standard Model.
\end{abstract}

\section{Introduction}

The new preprint [1] proposes contributions to several open problems in physics like the reconciliation of General Relativity (GR) with Quantum Physics, explaining the origin of gravity proposed as emerging from quantum (EPREinstein Podolsky Rosen) entanglement between particles, detailing contributions to dark matter and dark energy and explaining other Standard Model mysteries without requiring New Physics beyond the Standard Model other than the addition of gravity to the Standard Model Lagrangian. All this is achieved in a multi-fold universe that may well model our real universe, which remains to be validated.

With the proposed model of [1], spacetime and Physics are modeled from Planck scales to quantum and macroscopic scales and semi classical approaches appear valid till very small scales. In [1], it is argued that spacetime is discrete, with a random walk-based fractal structure, fractional and noncommutative at, and above Planck scales (with a 2-D behavior and Lorentz invariance preserved by random walks till the early moments of the universe). Spacetime results from past random walks of particles. Spacetime locations and particles can be modeled as microscopic black holes (Schwarzschild for photons and spacetime coordinates, and metrics between Reisner Nordstrom [2] and Kerr Newman [3] for massive and possibly charged particles - the latter being possibly extremal). Although surprising, [1] recovers results consistent with other like [4], while also being able to justify the initial assumptions of black holes from the gravity or entanglement model. The resulting gravity model recovers General Relativity at larger scale, as a 4-D process, with massless gravity, but also with massive gravity components

\footnotetext{
${ }^{1}$ shmaes.physics@gmail.com
} 
at very small scale that make gravity significant these scales. Semi-classical models also work well till way smaller scales that usually expected.

In this paper, we remain at a high level of discussion of the analysis and references are generic for the subjects. It makes the points accessible to a wider audience and keeps the door open to further papers or discussions devoted to details of interest. Yet, it requires the reader to review [1], as we do not revisit here all the details of the multifold mechanism or reconstruction of spacetime. More targeted references for all the material discussed here are compiled in [1].

\section{2. $\mathrm{SM}_{\mathrm{G}}$ : The Standard Model with Multi-Fold Gravity}

[1] proposes that in a multi-fold universe, the Lagrangian is complemented by terms associated to gravity and entanglement (in the form of the sum of the attractive effective potentials) [1].

$$
\mathscr{L}_{\text {total }}=\mathscr{L}_{S M}+\mathscr{L}_{\text {Gravity-with }-M F}+\mathscr{L}_{\text {EPR }}+\mathscr{L}_{\text {Others... }}
$$

The effect of gravity can be seen through the attractive potential contributions of all the energy sources. It can also been seen as expressing the Standard Model Lagrangian in curved spacetime (semi-classical point of view), now considered valid till small scales.

EPR entanglement is not believed to often play a significant role, except in dark matter use cases [5].

The last term is all other "New Physics" terms and we will consider it to be null.

With (1), the mass generation terms now involves the gravity effective potential described in [1]:

$$
\begin{aligned}
\mathscr{L}_{\text {int }} & \propto C_{1}(\text { vertex }) \\
& +m_{\text {acquired }}\left(1+\frac{V_{\text {eff }}}{m_{\text {acquired }}}\right)\left(C_{2}(\text { lepton } / \text { quark })\right. \\
& +C C)
\end{aligned}
$$

Where, the $C_{1}$ () designates the vertex contribution that represents the interaction with the Higgs/QCD Vacuum and/or Quark Gluon condensate with chirality flip and $C_{2}$ () represents the contributions of the right-handed + lefthanded leptons or quarks.

\section{Electroweak vacuum stability}

Since the work of Coleman [6,7], it is known that vacuum may not be unique in the universe. If a lower energy vacuum state exists, even separated with a high potential barrier, quantum tunneling implies that at some point, somewhere, the vacuum may switch to that truer vacuum $[8,9]$. In that case, even if with a very low probability, a bubble of nothing is expected to grow from that point, racing to ultimately annihilate (reconfigure) the whole part of the universe that it can reach as it expands.

This is rather a scary thought, and the risk of this happening warrants at least evaluating if this may actually happen to our universe. 
Since the discovery of the Higgs boson, the problem has been intensively studied [10]. The conclusion is that the Electroweak vacuum is stable but at the edge of instability. A puzzling result, that at the minimum warrants questioning why the Higgs mass would just be at such a value.

\section{Thank God for Gravity!}

[10] showed how the Electroweak vacuum stability depends on the mass of the top quark, which is the particle interacting with the strongest interaction with the Higgs Boson, along with the $Z$ boson.

When considering $\mathrm{SM}_{\mathrm{G}}$, we have:

$$
\mathscr{L}_{\text {int }} \propto m_{\text {Fermion }}+V_{\text {eff }}(\text { gravity })+V_{\text {eff }}(\text { EPR })
$$

So we see that the gravity effective potential from [1], $V_{\text {eff, }}$ can contribute the same way the mass top quark ( $m_{t}$ for $\left.m_{\text {Fermion }}\right)$ which is the dominant contribution to the Lagrangian. The other particles masses and the entanglement contributions are way smaller.

This means that the actual rest mass of the top quark is lower that estimated in the Standard Model without gravity. This shift of the stabilizes the Electroweak vacuum and in fact ensures that it is a stable and true vacuum as show with the graphs in [10].

In this example, we use gravity to change the fermion mass estimate without changing the overall impact of the Standard Model, where numerical estimates are well established. It is analogous in reasoning with the approach we used to resolve the strong CP violation problem [18].

The result is achieved within the context of the Standard Model with Gravity, in a multi-fold universe, and without the need of New Physics. For us we take this statements to refer to other symmetries (e.g. Supersymmetry) or Physics (e.g. Superstrings); at the difference of adding gravity as we propose.

Also, this analysis is for a Multi-fold universe as in [1]. [1] details arguments and ways to check its relationship with the real universe. Besides properties that can be experimentally verified (in the future because of the macroscopic weakness of gravity and gravity like effects for entangled systems), [1] shows how the multi-fold mechanisms and behaviors are in many aspects in today's conventional physics, that, at times, anticipates the behaviors modeled of a multi-fold universe. In addition, [1] explains many results obtained in gravity, quantum mechanics, General Relativity, superstring theory, Loop Quantum Gravity and the AdS/CFT correspondence conjecture. All these works attempt to come up with models for the real universe. It is at least a good sign that [1] may provide an interesting model of the real universe.

Other theories showing that gravity is relevant at the level of the standard model, can repeat the arguments, even with no relation to multi-fold universe and mechanisms or to gravity emergence from entanglement. So our model here is generic: if we add gravity to Standard Model with a model keeping it non negligible at the Standard Model scales, then there will be 3 and only 3 generations of Fermions.

If our model here is not validated by experience, it would not invalidate the multi-fold mechanism and the proposal that gravity emerges from entanglement as detailed in [1]. The analysis builds on [1], as a consequence of it, but it is not a condition for validation of multi-fold universes.

\section{Conclusions}


We believe that [1] makes a compelling case for the consistency of its multi-fold proposal. The present paper shows how the mechanisms of multi-fold universes can predict a stable true Electroweak vacuum; something that was much shakier and puzzling for the Standard Model without gravity.

The model works for multi-fold universe as well as in any situation where gravity is non negligible and added to the Standard Model.

This along with similar results in [1] and [5,11-17], make a strong case for more seriously considering the implications of adding gravity to the Standard Model to obtain $\mathrm{SM}_{\mathrm{G}}$, as a way to contribute to addressing open issues and offer better alternatives to New Physics speculations. This goes hand in hand with recognizing that this also implies the need to seriously consider that gravity may not always be negligible at the Standard Model scales as proposed in [1].

References: (most references come from popular science to make the discussion more approachable) [1]: Stephane H. Maes, (2020) "Quantum Gravity Emergence from Entanglement in a Multi-Fold Universe", viXra:2006.0088v1, (June 9, 2020).

[2]: https://en.wikipedia.org/wiki/Reissner\%E2\%80\%93Nordstr\%C3\%B6m metric

[3]: https://en.wikipedia.org/wiki/Kerr-Newman metric

[4]: Burinskii, Alexander, (2008), "The Dirac-Kerr-Newman electron", arXiv:0507109v4

[5]: Stephane H Maes, (2020), "Explaining Dark Matter Without New

Physics?", viXra:2007.0006v1, https://shmaesphysics.wordpress.com/2020/06/19/explaining-dark-energy-smallcosmological-constant-and-inflation-without-new-physics/, June 21, 2020.

[6]: Sidney Coleman, (1977), "Fate of the false vacuum: Semiclassical theory", Phys. Rev. D 15, 2929

[7]: Curtis G. Callan, Jr. and Sidney Coleman, (1977), "Fate of the false vacuum. II. First quantum corrections", Phys. Rev. D 16, 1762

[8]: The reference frame, (2011), "Bubble of nothing and other catastrophes", https://motls.blogspot.com/2011/10/bubble-of-nothing-and-other.html, Retrieved on April 14, 2020.

[9]: https://en.wikipedia.org/wiki/False vacuum.

[10]: J.R. Espinosa, G. Giudice, A. Riotto, (2017), "Cosmological implications of the Higgs mass measurement", arXiv:0710.2484v1.

[11]: Stephane H Maes, (2020), "Right-handed neutrinos? Mass? Ask Gravity", viXra:2007.0018v1, https://shmaesphysics.wordpress.com/2020/06/21/right-handed-neutrinos-ask-gravity/, June 21, 2020.

[12]: Stephane H Maes, (2020), "Explaining Dark Energy, Small Cosmological Constant and Inflation Without New Physics?", viXra:2006.0261v1, https://shmaesphysics.wordpress.com/2020/06/19/explaining-dark-energy-smallcosmological-constant-and-inflation-without-new-physics/, June 19, 2020.

[13]: Stephane H Maes, (2020), "Gravity Induced Anomalies Smearing in Standard Model so that Protons May Never Decay, Except in Black Holes ", viXra:2006.0128v1, https://shmaesphysics.wordpress.com/2020/06/12/protons-may-never-decay-except-in-black-holes/, June 13, 2020.

[14]: Stephane H Maes, (2020), "Gravity or Magnetic Monopoles? You Cannot Have Both!", viXra:2006.0190v1, https://shmaesphysics.wordpress.com/2020/06/15/gravity-or-magnetic-monopolesyou-cannot-have-both/, June 15, 2020.

[15]: Stephane H Maes, (2020), "Ultimate Unification: Gravity-led Democracy vs. UberSymmetries", viXra:2006.0211v1, https://shmaesphysics.wordpress.com/2020/06/16/ultimate-unification-gravityled-democracy-vs-uber-symmetries/, June 16, 2020.

[16]: Stephane H Maes, (2020), "Dualities or Analogies between Superstrings and Multi-fold Universe", viXra:2006.0178v1, https://shmaesphysics.wordpress.com/2020/06/14/dualities-or-analogies-betweensuperstrings-and-multi-fold-universes/, June 14, 2020. 
[17]: Stephane H Maes, (2020), “Gravity Dictates the Number of Fermion Generations:

3", viXra:2007.0068v1, https://shmaesphysics.wordpress.com/2020/06/24/gravity-dictates-the-number-offermion-generations-3/, June 24, 2020.

[18]: Stephane H Maes, (2020), "Strong CP Violation Tamed in The Presence of

Gravity", viXra:2007.0025v1, https://shmaesphysics.wordpress.com/2020/06/23/strong-cp-violation-tamed-inthe-presence-of-gravity/ , June 21, 2020. 